\title{
Electrical characterization of thin silicon films produced by metal-induced crystallization on insulating substrates by conductive AFM
}

\author{
Oxana Shamiryan*, Ivan Maidanchuk, Nari Ahn, Ilsang Choi and Ho Kyoon Chung \\ Samsung Mobile Display Co. Ltd, 508 Seongesong-dong, Seobuk-gu, Cheonan-si, Chungcheongnam-do, 330-300 Korea \\ *Oxana.Shamiryan@samsung.com
}

(Received: October 6, 2010; Accepted: February 20, 2011)

\begin{abstract}
We applied conductive Atomic Force Microscopy (c-AFM) to characterize the electrical properties of low-temperature polycrystalline silicon. Current distribution and current-voltage characteristics are recorded using conductive-diamond covered AFM tip and low noise external amplifier. Spectroscopic ellipsometry and optical microscopy are used to determine the grain size, crystalline fraction and film thickness. The correlation between structural properties of the poly-Si fabricated with varied conditions and c-AFM results is revealed, which shows the potential of this technique as convenient method for evaluation of metal-induced-crystallized poly-Si quality.
\end{abstract}

\section{Introduction}

Low-temperature polycrystalline silicon is one of the key materials for large area electronics on glass applications like active-matrix flat panel displays.[1] Metal-induced crystallization (MIC) method employing very low-dose crystallization catalyst, e.g. Ni, allows manufacturing of the poly-Si with super-large grains (Super Grain Silicon or SGS) for an active layer in top-gate thin film transistors (TFTs) on glass. As the poly-Si structure greatly influences electronic transport, grain size and crystalline $(c-\mathrm{Si})$ fraction were taken as an indication of the film quality.[2]

Crystallization process evaluation requires information about both long-range and short range non-uniformity. Spectroscopic ellipsometry is an effective method for long range uniformity analysis.[3] Relatively large light beam size (2x1 $\mathrm{mm}$ ) allows gathering information about crystallinity from area much larger than grain size. Evaluation of short-range non-uniformity caused by grain boundaries or metal defects, however, requires techniques with high spatial resolution, e.g. based on Scanning Probe Microscopy.

Most silicon studies by conductive Atomic Force Microscopy (c-AFM) involve thin films on conductive substrates, latter being necessary for current mapping of material with high amorphous content and small crystallite size. [4-7] However, Rezek et al. reported successful c-AFM mapping in an area $0.2 \mathrm{~mm}$ away from the bottom electrode, indicating that side-contact configuration might be possible.[8] The ability to use the method for thin films on insulating substrates is highly desirable for process monitoring, since fabrication of separate test glasses with bottom electrode is costly or simply not possible. We utilized conductive AFM as a tool for characterization of the quality of MIC SGS films on glass substrates.

\section{Experimental}

Amorphous silicon layers of fixed thickness were processed onto large glass substrates in a standard Plasma Enhanced Chemical Vapor Deposition (PECVD) system. Following a-Si deposition, capping $\mathrm{SiNx}$ layer and low dose (below $10^{14} \mathrm{at} / \mathrm{cm}^{2}$ ) of Ni catalyst were deposited. Crystallization process was carried out in the furnace at $650^{\circ} \mathrm{C}$ for 1 hour in nitrogen atmosphere. Varying plasma parameters and precursor gas dilution during $a$-Si deposition, samples with different grain size and crystallinity were obtained. Amorphous silicon films deposited with varied gas ratio were doped with boron $\left(2.5 \mathrm{e}^{12} \mathrm{at} / \mathrm{cm}^{2}, 10 \mathrm{keV}\right)$.

After crystallization, glass substrates were cut into $1 \mathrm{~cm} \times 1 \mathrm{~cm}$ samples. One set of samples was inspected by spectroscopic ellipsometry and subsequently mounted onto metal holders by conductive silver paste to provide an electric 
contact to poly-Si as illustrated in Fig. 1. An identical set of samples was used for the grain size measurements by optical microscopy after Secco etching to make grain boundaries visible. [10]

Simultaneous mapping of the topography and current has been done using XE-100 Scanning Probe Microscope from Park Systems corp. (Korea) with external linear low-noise amplifier capable to detect a current ranging from $1 \mathrm{pA}$ to $10 \mathrm{~mA}$

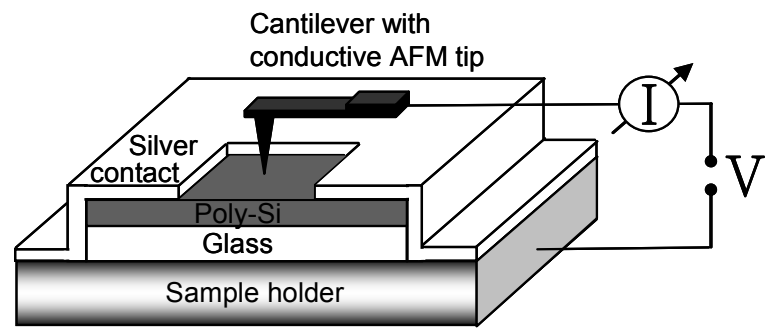

Fig. 1. Schematic cross-sectional representation of the sample structure and conductive AFM set-up.

We used silicon tips coated with highly doped polycrystalline diamond $(3-5 \mathrm{~m} \Omega \mathrm{cm}$ resistivity). As the relatively stiff cantilevers with force constant of $42 \mathrm{~N} / \mathrm{m}$ were used, the contact forces during scanning were maintained at $0.5 \mu \mathrm{N}$ level, providing a good electrical contact. The tip had been grounded and the DC bias was applied to the sample. Measurements were carried out at room temperature and $20 \%$ relative humidity.

To evaluate overall poly-Si conductivity and its uniformity across the scanned area, 16 current-voltage characteristics per sample were taken in a regular matrix, in the maximum bias interval from -10 to $+10 \mathrm{~V}$. All measurement parameters (sample geometry, grid size, contact force, time, etc.) were kept identical to enable comparison between different samples.

Crystallinity of poly-Si has been estimated by spectroscopic ellipsometry. $\Delta$ and $\Psi$ spectra were measured by J. A. Woollam M-2000 Spectral Ellipsometer in range $180-1800 \mathrm{~nm}$. Poly-Si was modeled as a mixture of amorphous and crystalline $\mathrm{Si}$ using Bruggemann Effective Medium Approximation. The fraction of crystalline silicon was extracted from fitting process. [2]

\section{Results and discussion}

\subsection{Local current mapping with $c-A F M$}

Characterization of the poly-Si in the lateral direction is important for evaluation of its performance in thin film transistors; however, this configuration decreases the detected current level in c-AFM compared to bottom-electrode arrangement due to the resistance of the film between the tip-surface contact point and edge contacts. Typical currents during scanning were in order of $0.1-1 \mathrm{nA}$. Tip-surface contact force and scan speed were selected so that no current degradation with time occurred, i.e. mean current level remained steady while the tip scanned along the same line for few minutes. Large scans (tens of micrometers) were found appropriate for both stable measurements and varied grain sizes that can be almost as large as the maximum possible scanning area of $50 \mu \mathrm{m} \times 50 \mu \mathrm{m}$. Since measurements were conducted in air, a thin oxide layer was present on silicon surface. It imposes an additional barrier for current flow; however, in our experiment the thin oxide film was partially abraded due to high contact force and use of the hard diamond tip. Surface oxide can be removed by short-time treatment in buffered oxide etchant (BOE) prior to the measurements, thereby improving the current images contrast.

\subsection{Poly-Si with varied grain size.}

For the first experiment, a set of four samples with varied grain sizes yet similar crystallinity was selected. The crystalline fraction of the samples estimated by ellipsometry is found to be $92.2 \pm 0.5 \%$; the grain size varied from $\sim 4 \mu \mathrm{m}$ to $\sim 25 \mu \mathrm{m}$. In Fig. 2, topography, current maps and optical images after Secco etching are shown for two samples with $\sim 6 \mu \mathrm{m}$ (a-c) and $\sim 25 \mu \mathrm{m}$ (d-f) average grain size. Topography represents smooth surfaces with root-mean-square (RMS) roughness below $1.5 \mathrm{~nm}$; no grain boundaries or grain-to grain thickness variations are visible on the surface without prior chemical treatment. However, current maps reveal contrasted areas of higher currents surrounded by lower-current regions (Fig. 2).

A typical low-dose MIC poly-Si film has a complex structure where grains are composed of 1) seed area, 2) radial growth region, and 3) high-angle grain boundaries (GBs) with neighboring grains. Within a single large grain, many sub-domains separated from adjacent sub-domains by low-angle grain boundaries are formed. Different regions (seed, growth and GB) have different crystallinity: the highest is in the growth region; the lowest is at the external GB and intermediate is at the seed.[2] Observed current contrast areas show correlation with the grain size determined from Secco images. Both external and intra-grain boundaries are visualized on the current images; highest currents match up the 
central and growth regions. The surface and interface morphology of our poly-Si films are very similar, thus effect of film thickness variations on the measured current is minimal.
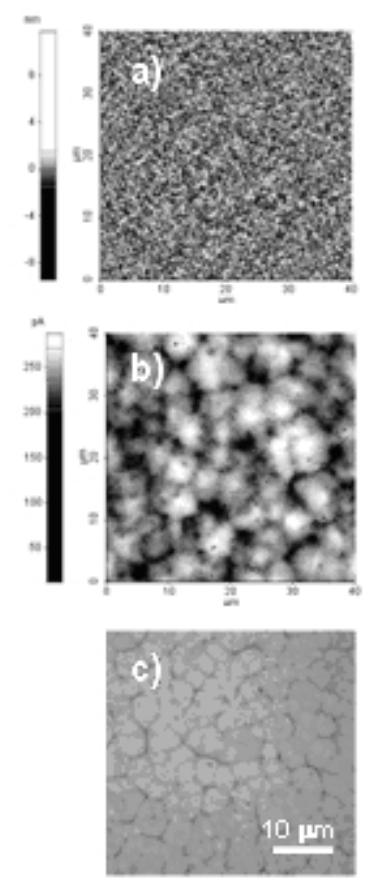
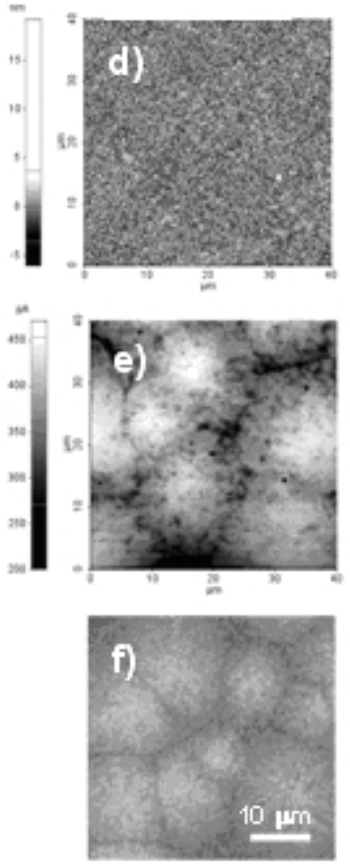

Fig. 2. Topography $(\mathrm{a}, \mathrm{d})$, corresponding current maps $(\mathrm{b}, \mathrm{e})$ and optical images after Secco etching $(\mathrm{c}, \mathrm{f})$ for two samples with different poly-Si grain size. Secco micrographs represent the typical film structure yet they were not taken from the same scan area.

Local current-voltage characteristics show non-Ohmic behavior. An example of I-V curves acquired on $40 \mu \mathrm{m} \times 40 \mu \mathrm{m}$ area is shown in Fig. 3 . For each tip position on the sample surface, the asymmetric I-V characteristics were recorded: higher currents were measured at negative voltages exceeding a threshold value; lower currents were observed at higher positive voltages. As the reverse part of the $\mathrm{I}-\mathrm{V}$ characteristics for voltages exceeding a barrier value can well be approximated by linear fit, we choose the $\mathrm{dI} / \mathrm{dV}$ at $-10 \mathrm{~V}$ as a representative parameter and plotted it versus average grain size for each sample (Fig. 4).

Since each grain boundary represent a potential barrier for the charge carriers, decrease in grain size and therefore increase in number of the GB is expected to result in higher overall film resistivity and lower $\mathrm{dI} / \mathrm{dV}$, as seen in Fig. 4. One can also notice that spread in the $\mathrm{dI} / \mathrm{dV}$ values is higher for larger-grain samples. These super-large grains are formed from few initial nuclei; random locations of these nuclei result in wide distribution in grain size. Therefore, local electrical properties of poly-Si are determined by highly non-uniform number and angle of grain boundaries for given direction. In contrast, high density of crystallization seeds per unit area produces smaller grains of similar size with large number of randomly orientated grain boundaries. Thus, proximity, size and direction of few large grain boundaries has a stronger influence on the current detected in each location compared to averaged effect of numerous smaller GBs.

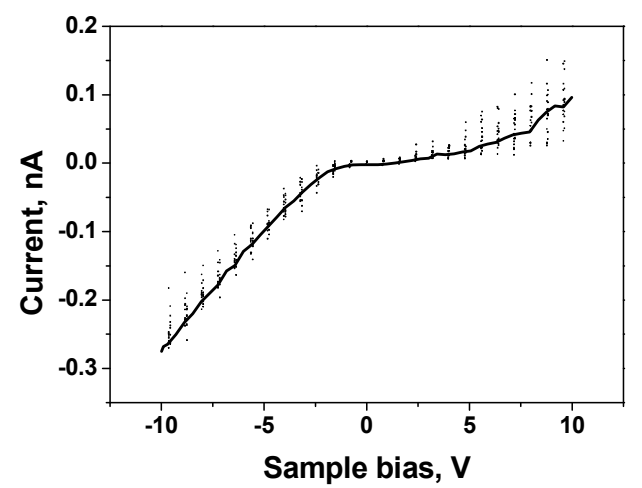

Fig. 3. A family of I-V curves taken in a regular matrix on one sample (dots) and averaged I-V curve (solid line). Only 10\% of data points are shown.

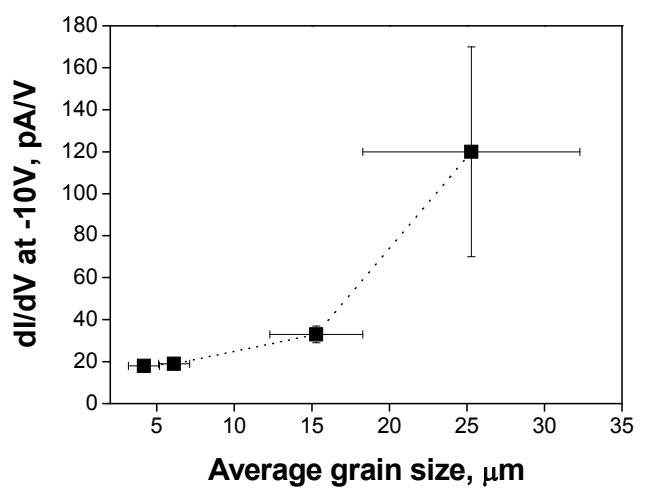

Fig. 4. Average dI/dV versus grain size for MIC samples with various grain size and similar crystallinity (c-Si fraction of $92.2 \pm 0.5 \%)$.

\subsection{Poly-Si with varied a-Si precursor}

The second set of four samples with varied $\mathrm{H}_{2}: \mathrm{SiH}_{4}$ ratio $(5: 1 ; 10: 1,20: 1$ and $40: 1)$ during a-Si deposition has been analyzed in the same experimental set-up. Secco images revealed average grain size of $\sim 11 \mu \mathrm{m}$ for lowest ratio (5:1) and poorly defined small-grain structure in the rest of the samples. Crystalline fraction of $92 \%$ for $5: 1$ ratio drops drastically to $62 \%$ for sample with high hydrogen content (40:1).

Topography images and current maps recorded on these samples show no distinctive features except for the typical small-scale roughness of the polysilicon surface with RMS of 1.6-2.0 nm (not shown). The current levels during the scanning 
were in order of $10 \mathrm{pA}-0.3 \mathrm{nA}$ at $-10 \mathrm{~V}$, with lowest value observed for the sample grown with highest hydrogen dilution. Typical I-V curves are shown in Fig. 5. Average grain size, c-Si fraction and $\mathrm{dI} / \mathrm{dV}$ for these samples are shown in Fig. 6.

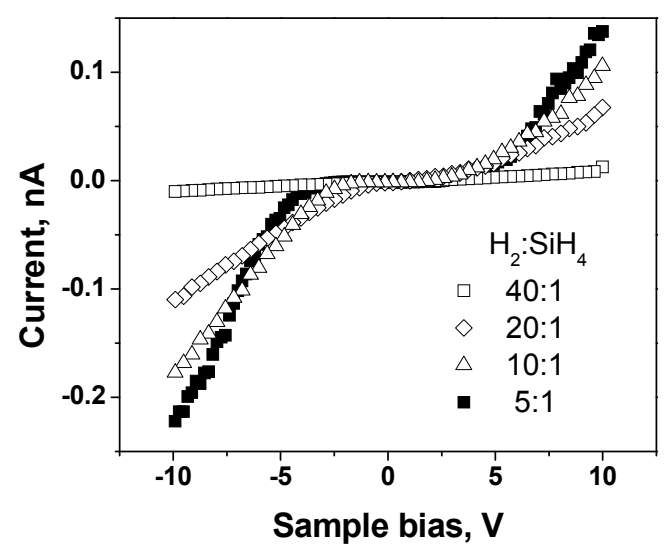

Fig. 5. Current-voltage characteristics of the MIC polysilicon films fabricated from a-Si with varied precursor gas ratios.

One may qualitatively explain the observed dependence considering the effect of the microstructural details of the $a$-Si precursor on MIC process and resulting poly-Si. As both crystalline fraction and grain size are varied in these samples, conductivity of the polysilicon and currents measured by c-AFM are the consequence of combination of these factors. In their turn, crystallization advance and grain size depend on the degree of order/disorder in the initial a-Si, while other process parameters, such as Ni dose, temperature and time being the same. Observed decrease in grain size and conductivity with the increase of hydrogen/silane ratio indicates a higher degree of disorder in $a$-Si and transition from metal-induced to solid-phase crystallization mechanism.

Further study is needed for comprehensive analysis of the conduction process in side-contact c-AFM configuration. The relation of the measured currents to the macroscopic conductivity and crystallinity of the poly-Si will be the subject of the future work.

\section{Conclusions}

We applied conductive AFM method for characterization of large-grain MIC polysilicon on glass substrates and successfully measured topography and local current under ambient conditions. C-AFM revealed the role of external and intra-grain boundaries as areas of lower currents. Observed correlation between structural properties of the poly-Si films and local currents detected by c-AFM shows a potential of the method to be used in a poly-Si quality monitoring.

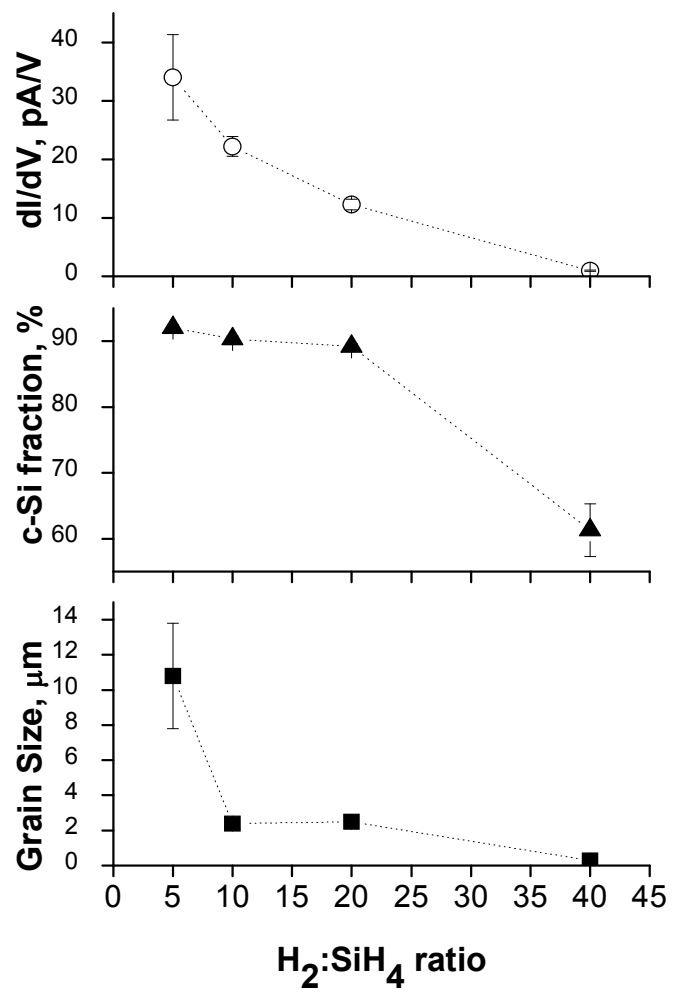

Fig. 6. Average grain size, I-V slope and crystalline fraction of the MIC polysilicon films fabricated from a-Si with varied precursor gas ratios.

\section{References}

[1] Yue Kuo, "Thin film transistors. Volume II: polycrystalline thin film transistors", Kluwer A P., p.2 (2004).

[2] Hyun-Woo Koo et al., International Meeting on Informational Display Digest, 20-2 (2009).

[3] P. Petrik, T. Lohner, M. Fried, L. P. Biró, N. Q. Khánh, J. Gyulai, W. Lehnert, C. Schneider, and H. Ryssel, J. Appl. Phys. 87, 1734 (2000).

[4] B.Rezek, J Stuchlík, A. Fejfar, J.Kocka, J. Appl. Phys. 92, 587 (2002).

[5] T. Fuyuki, T. Sameshima, H.P. Strunk and J.H. Werner, Solid State Phenomena 93, 339 (2003).

[6] R. Germanicus, E. Picard, B. Domenges, K. Danilo, K. Roger, Appl. Surf. Sci. 253, 6006 (2007).

[7] S. Honda et al., Thin Solid Films 501, 144 (2006).

[8] B. Rezek, J. Stuchlík, A. Fejfar, and J. Kočka, J. Appl. Phys. 92, 587 (2002).

[9] T. Mates, P.C.P. Bronsveld, A. Fejfar, B. Rezek, J. Koc`ka, J.K. Rath, R.E.I. Schropp, Journal of Non-Crystalline Solids 352, 1011 (2006).

[10] F. Secco d'Aragona, J. Electrochem. Soc. 119, 948 (1972). 